[0212-7199 (2004) 21: 10; pp 507-513] ANALES DE MEDICINA INTERNA Copyright (C) 2004 ARAN EDICIONES, S.L.

AN. Med. InTERnA (Madrid) Vol. 21, N. $^{\circ} 10$, pp. $507-513,2004$

\title{
Mujer de 41 años con dolor abdominal y fiebre de 24 horas de evolución
}

\author{
J. M. RAMOS, A. TORROBA ${ }^{1}$, J. M. GARCÍA SANTOS², M. C. MARÍN ${ }^{3}$ \\ Unidad de Enfermedades Infecciosas. Servicio de Medicina Interna. Hospital General

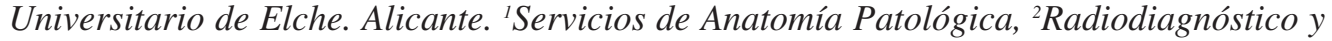 \\ de ${ }^{3}$ Medicina Interna. Hospital General Morales Meseguer. Murcia
}

\author{
A 41 YEAR-OLD FEMALE WITH ABDOMINAL PAIN AND FEVER OF \\ 24 HOURS
}

\section{RESUMEN}

Mujer de 41 años portadora de un dispositivo intrauterino (DIU) desde hacía 8 años que presentaba fiebre y dolor abdominal de 24 horas de evolución con leucocitosis. La radiología ponía de manifiesto engrosamiento del ciego, apéndice y ovario derecho con edematización de la grasa adyacente. En el diagnóstico diferencial hay que plantearse un problema digestivo como la apendicitis, la diverticulitis, la enfermedad inflamatoria digestiva, o la adenitis mesenterial, o pélvico ginecológico como la neoplasia óvarica, la endometriosis, el embarazo ectópico, la torsión ovárica y la infección de la pelvis. De las infecciones de la pelvis destacaría la enfermedad pélvico inflamatoria, la tuberculosis genital y la actinomicosis. Los antecedentes de DIU, la clínica y los hallazgos radiológicos como la presencia de una masa pélvica con invasión y engrosamiento de otras estructuras y la ausencia de adenopatías; hace más probable el diagnóstico de la actinomicosis abdominopélvica y como segunda posibilidad la tuberculosis genital.

PALABRAS CLAVE: Actinomicosis abdominopélvica. Enfermedad pélvica inflamatoria. Tuberculosis genital.

\begin{abstract}
A 41 year-old female with intrauterine contraceptive device (ICD) from 8 year ago that complained fever and abdominal pain during 24 hours and leucocitosis. The radiology examination tests showed cegal, appendiceal, and right ovary enlargement with swollen of fat adjacent. In the differential diagnosis should be include: apendicitis, diverticulitis, chronic inflammatory digestive disease or mesenterical adenitis. In the differential diagnosis included: ovarian neoplasm, endometriosis, ectopic pregnancy, ovarian torsion and pelvic inflammatory diseases. From pelvic infections, it is import consider pelvic inflammatory disease, genital tuberculosis and pelvic actinomycosis. With the antecedent of ICD, the clinic and the radiological finding as abdominal mass with invasion of adjacent structures and absence of adenopathy; the first diagnosis is a abdominopelvic actinomycosis and the second is a genital tuberculosis.
\end{abstract}

KEY WORDS: Abdominopelvic actinomycosis. Genital tuberculosis. Pelvic inflammatory disease.

Ramos JM, Torroba A, García Santos JM, Marín MC. Mujer de 41 años con dolor abdominal y fiebre de 24 horas de evolución. An Med Interna (Madrid) 2004; 21: 507-513.

\section{PRESENTACIÓN}

Mujer de 41 años que en las 24 horas previas a su ingreso presentó dolor abdominal difuso de tipo continuo con exacerbaciones, de intensidad variable no acompañadas de náuseas ni vómitos, y sí de dos deposiciones diarreicas de consistencia líquida. No refería ningún otro síntoma acompañante. La paciente era fumadora de 20 cigarrillos al día, tenía ciclos menstruales normales y era portadora de un dispositivo intrauterino (DIU) desde hacía 8 años, no se realizó revisiones en los últimos 5 años. Había tenido dos embarazos a término con gestaciones normales.

En el servicio de urgencia, la exploración física puso de manifiesto: presión arterial de 125/70 mm Hg y temperatura axilar de $38^{\circ} \mathrm{C}$. El estado general estaba afectado y mostraba palidez mucocutánea. En la exploración abdominal presentaba un abdomen doloroso difusamente, especialmente en hipogastrio sin signos de peritonismo. La puño percusión renal bilateral fue negativa. La exploración del aparato cardiorespiratorio y respiratorio era normal. En el análisis practicados en urgencias destacaban: hemoglobina, $10,5 \mathrm{~g} / \mathrm{dl}$; hematocrito, $32 \%$; VCM, $81 \mathrm{fl}$; leucocitos $20.600 / \mathrm{mm}^{3}$ (91\% segmentados, $5 \%$ linfocitos y $4 \%$ monocitos); plaquetas, $578.000 / \mathrm{mm}^{3}$; glucemia, $124 \mathrm{mg} / \mathrm{dl}$; creatinina, 0,69 mg/dl; sodio, $130 \mathrm{mEq} / \mathrm{l}$; y potasio, $4 \mathrm{mEq} / \mathrm{l}$. El sedimento de orina mostraba: más de 100 leucocitos/campo; 5-10 eritrocitos/campo; moderadas bacterias y células epiteliales. La radiografía posterolateral de tórax y el electrocardiograma fueron normal. La radiografía de

\footnotetext{
Trabajo aceptado: 1 de julio de 2004
}

Correspondencia: José Manuel Ramos Rincón. Unidad de Enfermedades Infecciosas (Servicio de Medicina Interna). Hospital General Universitario Elche. Camí de 1'Almazara, 11.03203 Elx, Alicante. e-mail: jramosrincon@yahoo.es 
abdomen en supino mostraba: distensión y meteorización de asas de intestino delgado con presencia de gas en el colon incluyendo ampolla rectal; y en bipedestación, presentaba algunos niveles de asas de intestino delgado. En la ecografía abdominal centrada en la pelvis demostraba signos de engrosamiento del ciego; apéndice agrandado en contacto íntimo con el ovario derecho que estaba aumentado de volumen y que mostraba estructuras de gas en su interior. El ovario izquierdo era prominente y de contornos mal definidos. Con la ventana doppler color se apreciaba un flujo llamativo en ambos ovarios. La tomografía computarizada (TAC) aportaba a la ecografía unas venas ováricas de calibre aumentado, edematización de la grasa pélvica, y ganglios de pequeño tamaño retroperitoneales, pélvicos y mesentéricos de dudosa significación.

A la vista del deterioro del estado general se practicó en mismo día de acudir a urgencias una exploración diagnóstica.

\section{DIAGNÓSTICO DIFERENCIAL}

Dr. JM Ramos: En resumen, se trata de una mujer de 41 años portadora de un DIU desde hacía 8 años que presentaba fiebre y dolor abdominal difuso especialmente en hipogastrio de 24 horas de evolución con leucocitosis, discreta anemia con tendencia a la microcitosis y trombocitosis. En la exploración radiológica puso de manifiesto niveles hidroáreos de intestino delgado, engrosamiento del ciego, apéndice y ovario derecho con burbujas de aire en su interior y edematización de las grasa adyacente. Desconocemos si se obtuvieron hemocultivos y urocultivos y el resultado de los mismos. También desconocemos si se realizó una exploración ginecológica y el resultado de la misma. A continuación rogaría que el radiólogo comentará con mayor detalle estos hallazgos radiológicos.

Dr. JM. García: En la ecografía abdominal sobre la pelvis demostraba signos de engrosamiento del ciego del que partía una estructura que parecía corresponder al apéndice de aspecto patológico. El apéndice mostraba un íntimo contacto con el ovario derecho que estaba aumentado de volumen, y que mostraba signos de gas en su interior (Fig. 1). El ovario izquierdo era prominente y mostraba al menos un quiste llamativo y unos contornos mal definidos. Con la ventana doppler color se apreciaba un flujo llamativo en ambos ovarios (Fig. 2). Se detectó la existencia de líquido libre intraperitoneal. En la tomografía computarizada (TAC) mostraba unas venas ováricas de calibre aumentado, edematización de la grasa pélvica, ganglios de pequeño tamaño retroperitoneales, pélvicos $\mathrm{y}$ mesentéricos de dudosa significación (Fig. 3). Los ovarios estaban aumentados de tamaño, con imágenes sugestivas de focos quísticos o necróticos en su interior, y gas evidente en el ovario derecho. La grasa pélvica mostraba una infiltración difusa, con imágenes densas lineales, así como líquido libre intraperitoneal, todo ello sugestivo de un proceso infiltrativo, probablemente inflamatorio con una pelviperitonitis, aunque no fue posible descartar que el origen fuera neoplásico.

Dr. JM. Ramos: En este caso destaca que la paciente era portadora de un DIU desde hacía 8 años. Y nos gustaría primeramente comentar ciertos aspectos de los DIU que podrían tener interés en este caso clínico. Los DIU son implantes que se emplean para la prevención del embarazo (1). Se disponen de dos tipos de dispositivos en España: el DIU de cobre y, recientemente, el DIU que libera levonorgestrel. Se han

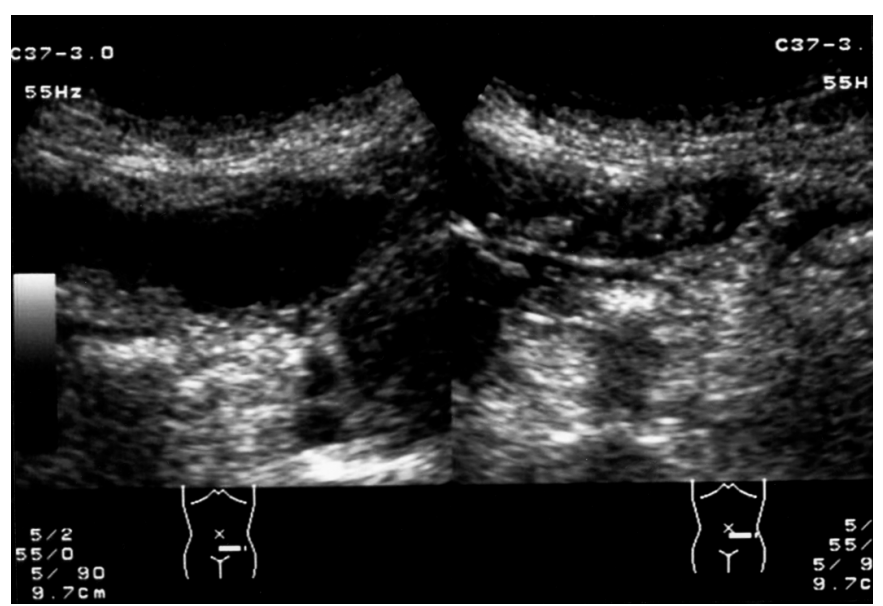

Fig. 1. Ecografía abdominal. Se observa un ovario derecho aumentado de volumen con gas en su interior.

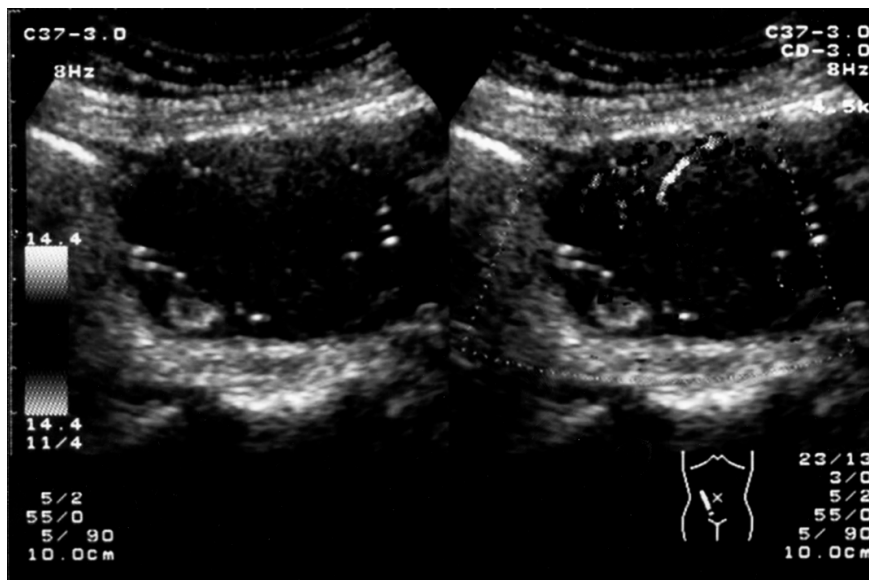

Fig. 2. Ecografía abdominal con doppler. Se observa una proliferación de estructuras vasculares en el ovario derecho, asociadas al aumento de tamaño y la presencia de cavidades quísticas y gas.

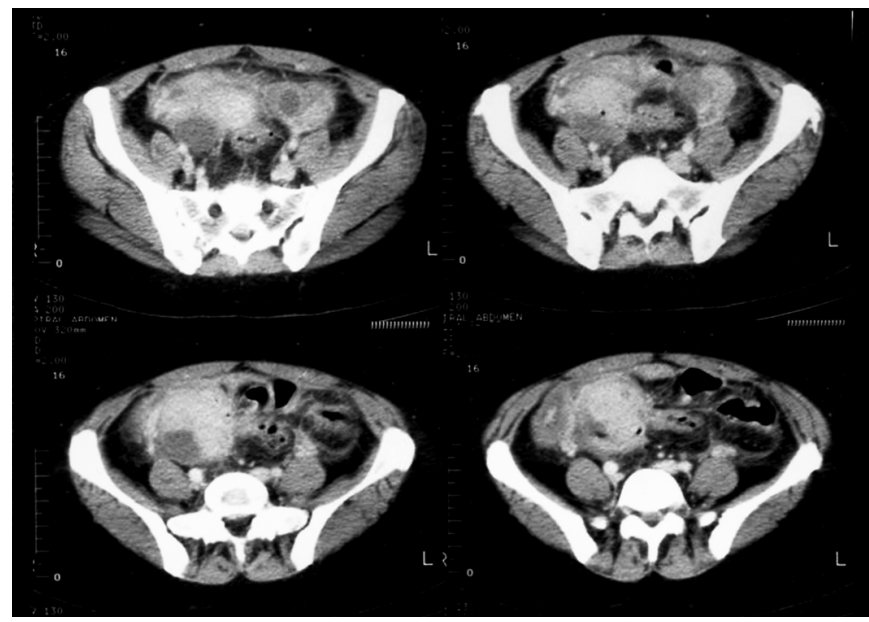

Fig. 3. Tomografía computarizada pélvica. Se observan edematización de la grasa pélvica, aumento de tamaño de los ovarios con focos quísticos o necróticos en su interior, y gas evidente en el ovario derecho

empleado dispositivos de plástico o de cobre sin estructura, que no están disponibles en la actualidad. En general se recomienda una revisión postinserción y posteriormente cada año hasta la retirada a los 5 años $(1,2)$. Los problemas de los DIU 
a largo plazo más importantes son: el embarazo, el embarazo ectópico (menos frecuente con el DIU de levonorgestrel), la hemorragia vaginal, la pérdida de las cuerdas de la cola y la enfermedad pélvica inflamatoria (EPI) (1). En la actualidad con los DIU de levonorgestrel el riesgo de EPI ha disminuido hasta alcanzar una tasa de 0,2 casos por 1000 mujeres-año (2). El DIU propicia la colonización cervical por actinomices (menos frecuente con el DIU de levonorgestrel) (3). No obstante la lesión de la parte alta del aparato genital es extremadamente rara.

Tras comentar el papel del DIU nos gustaría analizar la evolución de cuadro. Aunque la duración fue de 24 horas, pero teniendo en cuenta la anemia con tendencia a microcitosis y la trombocitosis, podría ser que la paciente llevara más días con el proceso, sin que le hubiera prestado atención. El principal hallazgo estaba en la pelvis y de forma práctica el problema abdominal podría tener su origen en la propia pelvis o ésta verse afectada secundariamente a enfermedades de estructuras próximas.

La rapidez del cuadro, de 24 horas, y la leucocitosis deben hacer pensar en una apendicitis; pero llama la atención la ausencia de síntomas digestivos, como nauseas o vómitos y de reacción peritoneal. En la ecografía realizada a la paciente se informa que el apéndice era patológico, si bien parece ser que estuviera afectada de la misma manera que otras estructuras adyacentes. En la TAC no se describen las imágenes características de una apendicitis aguda: apéndice anormal, inflamación periapendicular y cambios en la base del apéndice (4). Con los hallazgos de la clínica y de la radiografía parece descartar la apendicitis aguda como la causa del problema. De todas maneras el apéndice parece estar afectado secundariamente, por tanto no se puede excluir con rotundidad y cabría justificar la laparotomía exploradora.

La diverticulitis es una posibilidad teórica de inflamación pélvica abdominal; pero parece razonable eliminar este diagnóstico con las pruebas realizadas, ya que no se ha observado imágenes radiológicas de diverticulitis colónica en la TAC a pesar de la gran afectación de la pelvis. Otra enfermedad digestiva que pude afectar a la pelvis es la enfermedad de Crohn. Esta enfermedad inflamatoria afecta de forma parcheada a aparato digestivo y progresa englobando otras estructuras. Lo primero que se aprecia en la TAC es un engrosamiento del íleon terminal con o sin afectación de intestino delgado, colon o apéndice (5). En este caso se describe engrosamiento del colon, pero no se hace referencia al íleon terminal, y cabría esperar encontrarlo afectado, teniendo en cuenta la participación de otras estructuras pélvicas. Por ello en un principio permite descartarla. Otra posibilidad ante una cuadro de fiebre y dolor abdominal es la adenitis mesentérica por Yersinia enterocolitica y con menor frecuencia Yersinia pseudotuberculosis, pero la mínima afectación ganglionar en la TAC y la edad de la paciente nos haría descartar este diagnóstico.

Lo más probable es que la paciente tuviera un proceso primario de la pelvis y que las estructuras adyacentes que se ven afectadas en las pruebas de imagen lo fueran como consecuencia del problema pélvico inicial. Desde un punto de vista práctico los problemas del aparato genital de la paciente podrían agruparse en cinco apartados: las neoplasia benigna o maligna del ovario o trompa, la inflamación no tumoral (embarazo ectópico, endometriosis); los problemas mecánicos del ovario; los procesos metabólicos locales o la infección.

La imagen ecográfica de aumento de tamaño de los ova- rios, especialmente del derecho, debe hacer pensar en una neoplasia ovárica como primera posibilidad. Tingulstad et al (6) han propuesto el índice de malignidad, escala de riesgo de neoplasia ovárica de acuerdo con el estado menstrual, las características ecográficas y los niveles de CA 125; la presencia de una puntuación superior a 200 sugeriría una neoplasia ovárica, la sensibilidad de la escala es del 70-80\% y la especificidad del 92\%. Aunque no tenemos los niveles de CA 125, en el caso de que estuviera elevado, la puntuación estaría por debajo de 200 y ayudaría a descartar un proceso maligno. Pero continuaría indicada la realización de una citología o biopsia de la zona.

Un proceso local de la pelvis que podría cursar como en este caso es la endometriosis. Esta entidad consiste en la presencia de tejido muy semejante a la mucosa endometrial en lugares distintos del que fisiológicamente ocupa el endometrio. Existe una forma ovárica casi siempre bilateral y en la que aparece en forma de quiste y que puede romperse y formar densas adherencias con los órganos vecinos (7). Es raro que la endometriosis curse de forma tan aguda (24 horas) sin que la paciente no refiera ningunos de los síntomas cardinales de esta entidad: dismenorrea, menorragia y dispareunia. Eneste contexto, la endometriosis no parece aquí probable.

El embarazo ectópico o implantación del blastocisto fuera del endometrio del útero, es una complicación del DIU como se ha comentado previamente (8). El embarazo ectópico puede presentarse como en esta paciente con dolor abdominal y masa pélvica. Pero la presencia de la fiebre, leucocitosis elevada, trombocitosis y la rapidez del cuadro, a mi entender excluye esta posibilidad, si bien solicitaría una prueba de embarazo.

La torsión ovárica representa el 3\% de todas las urgencias ginecológicas (9), este caso corresponde con una urgencia ginecológica. Suele aparecer en jóvenes y las imágenes de la ecografía son características: folículos periféricos, alteración de la vascularización y aumento del tamaño del ovario (10). La ecografía y la TAC abdominal no muestran evidencias de esta enfermedad.

Desde un punto de vista teórico el síndrome de hiperestimulación ovárica como consecuencia de la terapia estimulante del ovario en el tratamiento de la infertilidad (11) podría incluirse en el diagnóstico diferencial de la paciente, pero la ausencia de este contexto clínico lo excluye.

Mead PB (12) divide las infecciones la pelvis de la mujer en tres apartados: las relacionadas con el parto, las que sucede tras una cirugía ginecológica y la EPI trasmitida sexualmente. Los dos primeros supuestos se descartan en este caso y por tanto quedaría la EPI. La EPI es una inflamación e infección de aparato genital femenino superior e incluye: la endometritis, la salpingitis, la pelviperitonitis y el absceso tubo-ovárico (13). Constituye un problema común de ingreso en la sala de ginecología de los hospitales norteamericanos. La morbilidad no es despreciable, y destaca: esterilidad, dolor pélvico crónico y embarazo ectópico. Los episodios repetidos de EPI cuadriplica o quintuplica el daño tubárico residual (13).

La mayoría de los cuadros ocurren como consecuencia de una infección ascendente a partir del cérvix. El daño inicial parece que esta causado por Neisseria gonorrhoeae y Chlamydia trachomatis, que permite la entrada posterior de otros microorganismos. Los aislamientos del tracto genital superior en la EPI suele ser polimicrobianos incluidos bacilos gramnegativos (enterobacterias), estreptococcos, Mycoplasma homi- 
nis y anaerobios (Peptostreptococcus spp, Bacteroides spp y Prevotella spp) (12,13).

Hay numerosos factores implicado en la EPI como: la historia de múltiples parejas sexuales, especialmente en los últimos 30días, la instrumentación del cérvix, la ducha vaginal (13) y la contracepción. El DIU se asocia con la EPI, con un riesgo calculado de presentar EPI en la mujeres con DIU entre un 0 y un 5\% (14). Durante la colocación del DIU podría existir una contaminación del endometrio lo que permite la aparición de la EPI aguda por microorganismo no implicados en la enfermedad transmisión sexual en los primeros 4 meses de la implantación (1).

El diagnostico clínico de la EPI es difícil, ya que los síntomas y signos clínicos son poco específicos. En 1998 se publicó los criterios de los Centers for Disease Control para el diagnóstico de la EPI (Tabla I). Una paciente para tener una EPI debe tener tres criterios mayores y un criterio menor (15). Con estos criterios diagnósticos el cuadro de la paciente es compatible con una EPI.

\section{TABLA I}

\section{CRITERIOS DIAGNÓSTICOS DE ENFERMEDAD PÉLVICA INFLAMATORIA}

Criterios mayores
Dolor abdominal bajo
Dolor a la movilización cervical
Inflamación ovárica bilateral

\section{Criterios menores}

Temperatura $>38,3^{\circ} \mathrm{C}$

Cervicitis mucopurulenta

Elevación de la VSG o PCR

Infección cervical por C. trachomatis o N. gonorrhoeae

La presencia de masa inflamatorio en la ecografía

En la EPI, la prueba diagnóstica de referencia es la laparoscopia (13). Se ha planteado otras pruebas de imagen en el diagnóstico como la ecografía transvaginal, el doppler color, TAC y más recientemente, la resonancia magnética (10, $13,16,17)$. El doppler color detecta cambios en el flujo de sangre asociado con la hiperemia debida a la inflamación tubárica con un valor predictivo positivo del $91 \%$ y negativo del $100 \%$ en la mujeres con y sin enfermedad tubárica (17). Me gustaría destacar en este momento que en la eco-doppler de la paciente se observaba un aumento del flujo sanguíneo en ambos ovarios y que las venas ováricas en la TC estaban dilatadas lo que nos acercaría al diagnóstico de una EPI. Otra técnica es la gammagrafía con leucocitos marcados con tecnecio (sensibilidad de $100 \%$ y especificidad del 92\%) (18), pero disponible en pocos hospitales.

La paciente llevaba 8 años con el DIU, por tanto una EPI clásica, aunque no se puede descartar, parece que no deba ser considerada en este momento, ya la EPI aparece en los primeros meses tras la implantación del DIU (13). Sin bien, un absceso tubo-ovárico con afectación de estructuras adyacentes por una flora polimicrobiana incluidos anaerobios que provocaría las burbujas en el ovario derecho visualizadas en la ecografía no puede excluirse.

Hay una forma de EPI aguda, que es similar a una apendi- citis; diagnosticada generalmente por laparotomía y que es el salpingitis por Streptococcus pneumoniae (19) o Haemophilus influenzae (20) consiste en una infección genital aguda adquirida por vía ascendente a partir de la vagina con afectación tubárica con o sin implicación ovárica. Esta infección parece ser más frecuente en las portadora de DIU, pese a su rareza. Debido a la afectación extensa visualizada en la TAC y ecografía hace que no deba ser considerada en este momento.

Quisiera citar rápidamente otras infecciones tubo-ováricas específicas, que desde un punto de vista teórico podría incluirse en el diagnostico diferencial de este paciente, pero que su infrecuencia y la ausencia de contexto clínico y epidemiológico adecuado hacen que no deban ser consideradas en este momento. Entre ellas cabe mencionar el absceso tubo-ovárico por Pseudomonas aeruginosa en portadora de DIU (21), el absceso tubo-ovárico por Brucella (22), la aspergilosis pélvica en inmunudeprimidas (23), el absceso tubo-ovarico por Coccidioidides imitis (24), la infestación pélvica por formas parasitarias de Enterovius vermicularis (25) o la hidatidosis tubo-ovárica (26). Todos estas infecciones fueron diagnosticadas en la laparotomía y no se habían sospechado previamente.

Otra rara enfermedad que puede provocar una marcada afectación del ovario, trompa y estructuras adyacentes es el absceso tubo-ovárico xantogranulomatoso (276). Esta enfermedad se ha visto en mujeres con EPI de repetición o con divertículos colónicos y se acompaña de imágenes quísticas del ovario. En esta paciente las manifestaciones clínicas son compatibles pero la ausencia de EPI previa o de divertículos de colon, a mi entender me permite no considerar esta rara posibilidad.

Una vez realizadas estas consideraciones acerca de la EPI, continuaré la discusión con las dos enfermedades inflamatorias pélvicas específicas que podría tener la paciente: la tuberculosis pélvica y la actinomicosis abdominopélvica asociada al DIU. La tuberculosis genital es un proceso raro en países industrializados (entre 1-5\%) y en España se diagnostica ocasionalmente y cada vez con menos frecuencia (28); a diferencia de los países en vías de desarrollo, donde es una causa común de EPI (29). La tuberculosis alcanza el aparato genital femenino generalmente por vía hematógena y anida inicialmente en el endosalpinx y posteriormente se extiende a endometrio, ovarios, cérvix y vagina. Con frecuencia la tuberculosis genital es asintomática o los síntomas son poco específicos $(30,31)$ como esterilidad, irregularidades mestruales y dolor abdominal (29). Otras veces se comporta como una EPI que no responde al tratamiento convencional.

Para Schaefer (32), la tuberculosis genital podría ser de dos tipos: la tuberculosis mínima, sin casi síntomas y sin masas anexiales y que representa el $90 \%$ y la tuberculosis avanzada con masa anexiales palpables. En este paciente el cuadro clínico de fiebre y la masa anexial de tamaño significativo en la ecografía es perfectamente compatible con una tuberculosis avanzada.

Para el diagnóstico de la tuberculosis genital puede ser de ayuda una prueba de Mantoux positiva y la existencia de otro foco tuberculoso en otro órgano como el pulmón, si bien la asociación es muy rara (30-32). Se puede recurrir para al diagnostico a la biopsia de endometrio para estudio histológico y cultivo, y en menos frecuencia al cultivo de secreciones uterinas y sangre mestrual (31). Pero la prueba complementaria que más ha ayudado para diagnosticar la tuberculosis genital 
ha sido la histerosalpingografía, con una imágenes del útero y trompas muy características (32). En la actualidad con la resonancia magnética se consiguen imágenes que permiten un acercamiento al diagnóstico (33).

En esta paciente con los datos que se disponen a favor de la tuberculosis estaría el aumento de tamaño de los ambos ovarios especialmente el derecho y la presencia de ganglios de pequeños tamaño retroperitoneales, pélvicos y mesentéricos. En contra estaría la implicación del ciego y el apéndice, que es poco común, si fuera así estaríamos ante una tuberculosis genital y abdominal y por tanto el peritoneo debería verse afectado en la ecografía y TAC con ascitis, engrosamiento y/o opacidades nodulares sugerentes de implantes peritoneales que no estaban presente en este caso (10). Otros datos que nos puede ayudar a excluirla, sería la falta da datos indirectos de tuberculosis actual o previa en la radiografía de tórax y la agudeza con la que se presentó el cuadro.

La actinomicosis es una infección granulomatosa supurativa, crónica que cursa con la formación de abscesos que se extienden formando trayectos fistulosos. La actinomicosis esta causada por actinomicetales, microorganismos grampositivos, anaerobios, que crecen lentamente formado filamentos ramificados y se reproducen por fragmentación y formación de esporas. Inicialmente se clasificaron como hongos, pero actualmente son consideradas bacterias. De las diferentes especies de actinomicetales el más frecuente es el Actinomyces israelii, seguido a distancia de Propionbacterium propionicum, Actinomyces naeslundii y Actinomyces odontolyticum (34) Estos microorganismos pueden afectar todos los órganos, pero se han descrito cinco tipos de infección: cervicofacial, cutánea primaria, torácica, gastrointestinal y pélvica (34).

En la actualidad existe una preocupación con la actinomicosis en usuarias del DIU (1). Entre el 4 y el $11 \%$ de las mujeres con DIU se ven en la citología vaginal gránulos de azufre o microorganismos compatibles con actinomices $(35,36)$; con un riesgo 2 a 4 veces superior respecto a las mujeres sin DIU $(37,38)$. La incidencia de colonización cervical por actinomices se incrementa con la duración del DIU (35). A pesar de la colonización vaginal el riesgo de afectación de la parte alta del aparato genital es extremadamente raro (1). El desarrollo de la enfermedad pélvica vendría desde la zona cervico vaginal como consecuencia de la erosión mecánica que causa el DIU en la mucosa uterina.

El primer caso de actinomicosis pélvica asociada a DIU fue notificado en 1930 (35). Desde entonces se han descrito al menos un centenar de casos (35), alguno en la literatura nacional $(40,41)$. Como en la colonización hay una relación entre el tiempo de implantación del DIU y el riesgo de actinomicosis pélvica; se recogen periodos variables, pero en general está entorno a los 5 años (35), como en el caso de la paciente que nos atañe. Esta enfermedad suele presentar un curso insidioso y produce síntomas inespecíficos como dolor en hipogastrio, polaquiruia, náuseas o febrícula $(35,40,41)$. La forma de presentación más común es el absceso tubo-ovárico uni o bilateral (42). En otras ocasiones puede presentarse como un absceso de pared abdominal, absceso glúteo, fístula vesicouterina, fístula ílio pélvica, fístula cutánea hidroureter, hidronefrosis, o piometra $(35,39-43)$. La infección asintomática es excepcional. Es muy raro (35) que la paciente acuda al servicio de urgencias por un dolor abdominal intenso como en este caso, en el que se plantea el diagnóstico de un abdomen agudo.

En esta paciente en el análisis de sangre tenía una anemia normocítica, leucocitosis y elevación de la VSG, como se ha visto en las mujeres con actinomicosis con DIU (35,3941,43,44). En el trabajo de Lee et al (45), donde revisan 18 casos de actinomicosis gastrointestinal, 8 eran en mujeres con DIU, describen que la actinomicosis pélvica suele aparecer como una masa pélvica o peritoneal con infiltración de estructuras adyacentes y engrosamiento de la paredes de intestino próximas al proceso. En este caso los hallazgos de la ecografía y la TAC eran más sugestivos de actinomicosis que de tuberculosis, ya que parece que la actinomicosis cursa con una afectación difusa y sin adenopatía, y las adenopatías irían más a favor de tuberculosis $(10,45)$. En este caso no se veían adenopatías o el propio radiólogo cuestionaba su relevancia. En mi opinión, la resonancia magnética hubiera aportado datos de actinomicosis como una masa hiperintensa en $\mathrm{T} 2$ en el útero, el parametrio y los ovarios, y elementos para descartar una neoplasia ovárica o genitourinaria (46). Por ultimo un comentario acerca de la lo adecuado de la punción guiada por la TAC para el diagnóstico citológico de actinomicosis (47), si bien en la mayoría de los casos comunicados en la literatura, el cuadro no es sospechado y generalmente se llega a la cirugía.

En definitiva, creo que en esta paciente el diagnóstico más probable es actinomicosis abdominopélvica en mujer portadora de DIU. Como segunda posibilidad estaría una tuberculosis pélvica. Para llegar al diagnóstico se podría haber realizado una citología vaginal y/o cultivo de los hilos del DIU; si bien la forma definitiva es obtener una muestra de tejido de la zona afectada, que en este caso sería el ovario derecho. En mi opinión el procedimiento diagnostico fue una laparotomía o una laparoscopia con toma de biopsia. Quisiera reseñar que en caso de existir una sospecha de actinomicosis por una citología compatible, la punción-aspiración con aguja fina dirigida por la ecografía o la TAC hubiera permitido llegar al diagnostico definitivo.

Dr. Soria: Se practicó una laparotomía de urgencia en la que se observó líquido libre seropurulento, plastrón inflamatorio que englobaba a útero, anejos, ciego, ileon y sigma. El ovario y trompa derecha tenían aproximadamente $12 \mathrm{~cm}$ de diámetro con claro aspecto inflamatorio. El apéndice mostraba signos inflmatorios. Se practicó una disección dificultosa del plastrón inflamatorio, una anexectomía derecha y apendicetomía sin poder invaginar el muñón apendicular por inflamación de la pared cecal. Se respetó el anejo izquierdo a pesar de los signos inflamatorios por tener aspecto viable.

\section{DIAGNÓSTICO DR. JM. RAMOS}

\section{ACTINOMICOSIS ABDOMINOPÉLVICA}

\section{Discusión anatomopatológica}

Dra. A. Torroba: Se reciben dos piezas quirúrgicas; una viene como ovario y la como apéndice cecal. En el ovario se observa casi total sustitución del parénquima ovárico normal por un denso infiltrado polimorfo dispuesto en forma de abscesos supurativos centrales, con tejido de granulación alrededor y fibrosis cortical que los delimitaba. En el seno de esos abscesos se reconocen unas formaciones redondeadas de tamaños variables, muy eosinófilas (Fig. 4) y de bordes espiculados, que se teñían intensamente con la técnica histoquímica del Gram (Fig. 5) y también con la del Groccott, lo que corresponde a bacterias grampositivas y ramificadas. Con la técnica del Groccott (Fig. 6) se obser- 


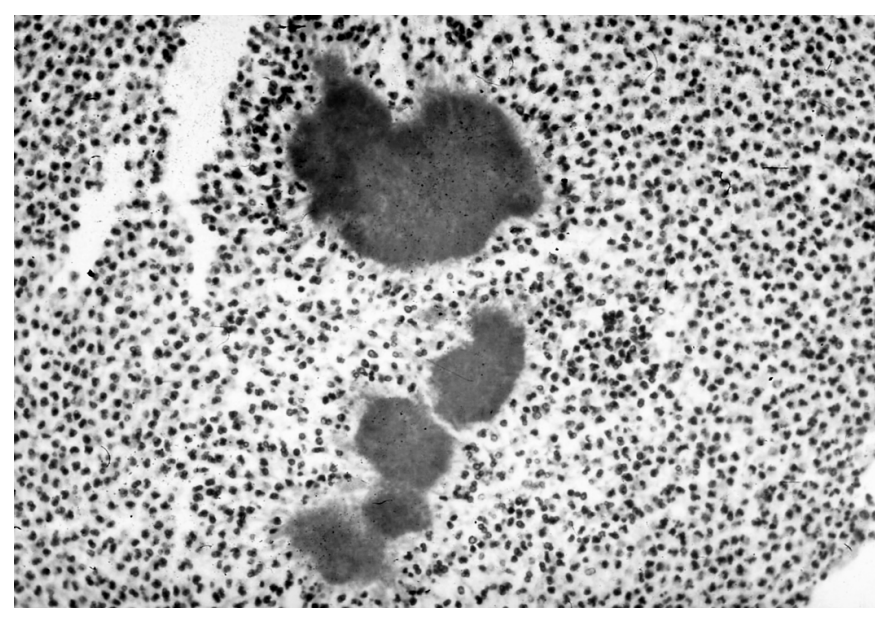

Fig. 4. Biopsia de ovario: infiltrado polimorfo dispuesto en forma de abscesos supurativos centrales (hematoxilina-eosina, x200).

vaban perfectamente los citados filamentos de estas bacterias. Adherida a la superficie ovárica se identificaba una pared tubárica con intensa inflamación crónica y presencia de abundantes leucocitos polinucleares intraluminales. El apéndice mostraba una pared apendicular con importante ampliación fibrosa y signos de periapendicitis.

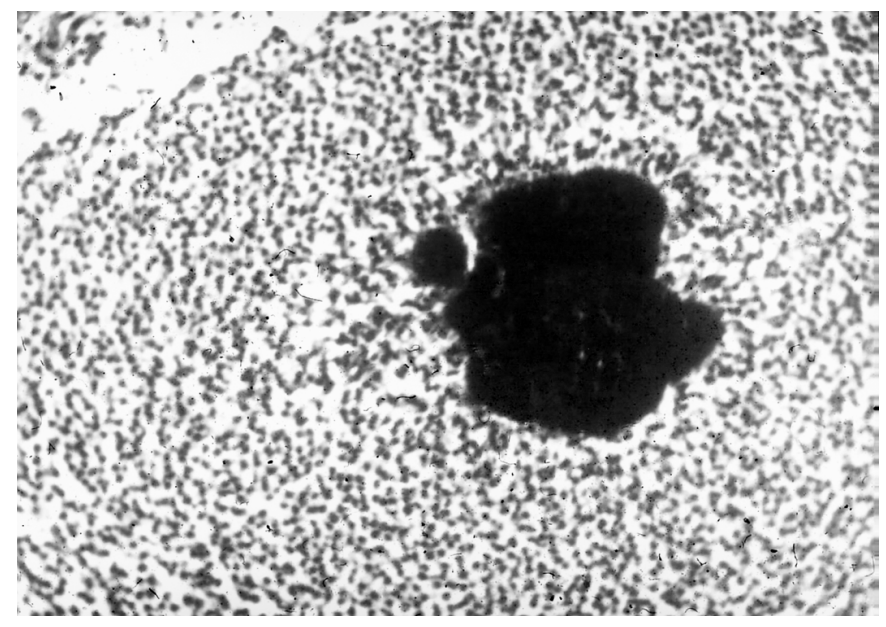

Fig. 5. Biopsia de ovario: abscesos grampositivos (tinción de Gram ×200).

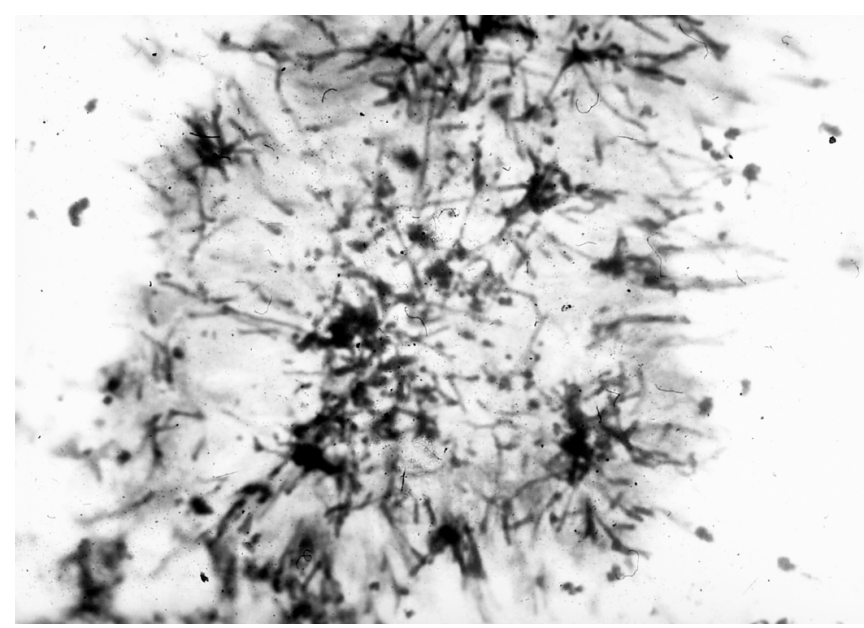

Fig. 6. Biopsia de ovario: bacterias ramificadas (tinción de Grocott x1000).

Dr. F. Herrero: ¿Cuál fue la evolución de la paciente?

Dr. V. Soria: La paciente fue operada de urgencias y permaneció en la planta de Cirugía General con clindamicina (600 mg cada 6 horas) y ciprofloxacina intravenosa (200 mg cada 12 horas). Mejoró progresivamente permaneciendo afebril. Al quinto día del postoperatorio se decidió su alta hospitalaria. Al onceavo día tras la intervención fue revisada en las consultas externas de cirugía. Tras recibir el resultado de anatomía patológica se remite a la paciente a la consulta de Enfermedades Infecciosas.

Dra. A. Mensalvas: La paciente fue tratada con amoxicilina 1 gramos cada 8 horas de forma oral con una buena evolución tras 1 año de seguimiento.

\section{AGRADECIMIENTOS}

Al Dr. V. Soria del Servicio de la Cirugía General y a la Dra. A. Menasalvas de la Unidad de Enfermedades Infecciosas, Servicio de Microbiología del Hospital Morales Meseguer por la aportación del caso clínico y su colaboración en la presentación. Al Dr. F. Herrero, del Servicio de Medicina Interna, por la coordinación de la sesión clínico patológica.

\section{Bibliografía}

1. Nelson AL. The intrauterine contraceptive device. Obstet Gynecol Clin North Am 2000; 27: 723-740.

2. Sivin I. Risks and benefits, advantages and disadvantages of levonorgestrel-releasing contraceptive implants. Drug Safety 26: 303-335.

3. Merki-Feld GS, Lebeda E, Hoog B, et al. The incidence of actinomyceslike organisms in Papanicolaou-stained smears of copper- and levonorgestrel-releasing intrauterine devices. Contraception 2000; 61: 365-368.

4. Rao PM, Muller PR. Clinical and pathologic variants of appendiceal disease: CT features. AJR 1998; 170: 1335-1340.
5. Allen DC, Calvert CH. Crohn's ileitis and salpingo-oophoritis. Ulster Med J 1995; 64: 95-97.

6. Tingulstad S, Hagen B, Skjeldestaol FE, et al. Evaluation of a risk of malignancy index based on serum CA125, ultrasound findings and menopausal status in pre-operative diagnosis of a pelvic mass. BJOG 1996; 103: 826-831.

7. Olive DL, Schwartz LB. Endometriosis. N Engl J Med 1993; 328: 1759-1769.

8. Meirik O, Farley TMM, Diaz S, et al. Post-marketing surveilance of Norplant contraceptive implants: I. Contraceptive efficacy and reproductive health. Contraceptive 2001; 63: 167-186. 
9. Houry D, Abbott JT. Ovarian torsion: a fifteen year review. Ann Emerg Med 2001; 38: 156-159.

10. Bennett GL, Harvey WB, Slywotzky CM, et al. CT of the acute abdomen: gynecologic etiologies. Abdom Imaging 2003; 28: 416-432.

11. Kim IY, Lee BH. Ovarian hyperstimulation syndrome: US and CT appearances. Clin Imaging 1997; 21: 284-286.

12. Mead PB. Infections of the female pelvis. En: Mandell, Douglas, and Bennett's Principles and Practice of Infectious Diseases, Mandell GL, Bennett JE, Dolin R (eds); $5^{\circ}$ ed. Nueva York: Churchill Livingstone, 2000; 1235-1243.

13. Beigi RH, Wiwsenfeld HC. Pelvic inflammatory disease: new diagnostic criteria and treatment. Obstet Gynecol Clin N Am 2003; 30: 777793.

14. Shelton JD. Risk of clinical pelvic inflammatory disease attributed to an intrauterine device. Lancet 2001; 357: 443.

15. Centers for Disease Control and Prevention. 1998 guidelines for treatment of sexually transmitted diseases MMWR Morb Mortal Wkly Rep 1998; 47: 1-116.

16. Molander P, Sjöberg J, Paavonen J, et al. Transvaginal power doppler findings in laparoscopically proven acute pelvic inflammatory disease. Ultrasound Obstet Gynecol 2001; 17: 233-238.

17. Ross JD. Pelvic inflammatory disease: how should it be managed? Curr Opin Infect Dis 2003; 16: 37-41

18. Rachinsky I, Boguslavsky L, Goldstein D, et al. Diagnosis of pyogenic pelvic inflammatory diseases by $99 \mathrm{~m}$ Tc-HHMPAO leukocyte scintigraphy. Eur J Nucl Med 2000; 27: 1774-1777.

19. De Celis G, Casl J, Pastora L. Absceso tubárico bilateral sin peritonitis por Streptococcus pneumoniae. Enferm Infecc Microbiol Clin 2000; 18 : 92-93.

20. Rodríguez-Guardado A, Alvarez M, Méndez Lage S, et al. Salpingitis bilateral por Haemophilus influenzae en una paciente sin factores de riesgo. Med Clin (Barc) 2000; 114: 758-759.

21. King JA, Olsen TG, Lim R. Pseudomonas aeruginosa-infected IUD associated with pelvic inflammatory disease. A case report. J Reprod Med 2002; 47: 1035-1037.

22. Seoud MA, Kanj SS, Habli M, et al. Brucella pelvic tubo-ovarian abscess mimicking a pelvic malignancy. Scand J Infect Dis 2003; 35: 277-278.

23. Kim SW, Nah MY, Yeam CH, et al. Pelvic aspergillosis with tubo-ovarian abscess in a renal transplant recipient. J Infect 2001; 42: 215-217.

24. Chowfin A, Tight R. Female genital coccidioidomycosis (FCG), Addison's disease and sigmoid loop abscess fue to Coccidioides immites; case report and review of literature on FGC. Mycopathologia 1999; 145: 121-126.

25. Das DK, Pathan SK, Hira PR, et al. Pelvic abscess from Enterobious vermicularis. Report of a case with cytologic detection of eggs and worms. Acta Cytol 2001; 45: 425-429.

26. Gaym A, Abebe D, Degefe DA. Hydatid cyst an unusual cause of ovarian enlargement. Ethiop Med J 2002; 40: 283-291.

27. Mesia AF, Lam H, Wallach RC. Xanthogranulomatous tubo-ovarian abscess resulting from chronic diverticulitis. Gynecol Obstet Invest 2000; 49: 70-72.

28. Nistal de Paz F, Herrero Fernández BB, Pérez Simón R, et al. Pelvic-peritoneal tuberculosis simulating ovarian carcinoma: report of three cases with elevation of the CA 125. Am J Gastroenterol 1996; 91: 1661-1662.
29. Namavar Jahromi B, Parsanezhad ME, Ghane-Shirazi R. Female genital tuberculosis and infertility. Int J Gynecol Obstet 2001; 75: 269-272

30. Hunter D, Sim D. Pelvic tuberculosis: a case of delayed diagnosis. Acta Obstet Gynecol Scand 2001; 80: 281-282.

31. Sinha P, Johnson AN, Chidamberan-Pillai S. Pelvic tuberculosis: an uncommon gynaecological problem presenting as ovarian mass. BJOG 2000; 107: 139-140.

32. Schaefer G. Tuberculosis of the female genital tract. En: Clinical Gynaecology; vol 1. Nueva York: Lippincott, 1991: 1-20.

33. Crowley JJ, Ramji FG, Amundson GM. Genital Tract tuberculosis with peritoneal involvement: MR appearance. Abdom Imaging 1997; 22 445-447.

34. Russo TA. Agents of actinomycosis. En: Mandell, Douglas, and Bennett's Principles and Practice of Infectious Diseases, Mandell GL, Bennett JE, Dolin R (eds); $5^{\circ}$ ed. Nueva York: Churchill Livingstone, 2000; 2645-2654

35. Fiorino AS. Intrauterine contraceptive device-associated actinomycotic abscess and Actinomycetes detection on cervical smear: Review. Obstet Gynecol 1996; 87: 142-149.

36. Garbin O, Hummel M, Camus E, Dellenbach P. [Pelvic actinomycosis: the value of preoperative diagnosis. Apropos of a case]. J Gynecol Obstet Biol Reprod (Paris) 1994; 23: 395-401.

37. Citron J, del Pino A, Duarte B, Wood D. Abdominal actinomycosis. Dis Colon Rectum 1996; 39: 105-108.

38. Catawani A, Soheil A. Incidence of actinomicosis associated with intrauterine devices. J Reprod Med 1994; 39: 585-587.

39. Nawroth F, Foth D, Schmidt T, et al. Differential diagnosis and non-surgical treatment of pelvic actinomycosis. Acta Obstet Gynecol Scand 2000; 79: 1024-1025.

40. Pérez García MD, Rodríguez Alonso A, Núñez López A, et al. Actinomicosis abdominopélvica con afectacion del aparato urinario, secundaria a infección ginecológica por dispositivo intrauterino. Acta Urol Esp 2000; 24: 197-2000.

41. Locutura J, Lorenzo JF, Miján A. Actinomicosis abdominopélvica en una portadora de dispositivo intrauterino: tratamiento con ceftriaxona. Enferm Infecc Microbiol Clin 1999; 27: 46-47.

42. Burkman R, Schlesselman S, McCaffrey L, et al. The relationship of genital tract actinomyces and the development of pelvic inflammatory disease. Am J Obstet Gynecol 1982; 143: 585-589.

43. Feiter PW, Soeters PB. Gastrointestinal actinomycosis: an unusual presentation with obstructive uropathy. Dis Colom Rectum 2001; 44 1521-1525.

44. Tedeschi A, Di Mezza G, D'Amico O, et al. A case of pelvic actinomycosis presenting as cutaneous fistula. Eur J Obstet Gynecol 2003; 108: 103-105.

45. Lee IJ, Ha HK, Park CM, et al. Abdominopelvic actinomycosis involving the gastrointestinal tract: CT features. Radiology 2001; 220: 76-80.

46. Hawnaur JM, Reynols K, McGettigan C. Magnetic resonance imaging of actinomycosis presenting as pelvic malignancy. Br J Radiol 1999; 72: 1006-1011.

47. Lee YC, Min D, Holcomb K, et al. Computed tomography guided core needle biopsy diagnosis of pelvic actinomycosis. Gynecol Oncol 2000 79: 318-323. 